\title{
Comportement de certains fruits tropicaux traités par déshydratation- imprégnation par immersion dans une solution de saccharose
}

\author{
Yvette Jiokap Nono ${ }^{a \star}$, Guy Bertin Nuadje ${ }^{a}$, Anne-Lucie Raoult-Wack ${ }^{b}$, François Giroux ${ }^{c}$
}

a Département de Génie des procédés et d'ingénierie, École nationale supérieure des sciences agro-industrielles (Ensai),

Université de Ngaoundéré, BP 455, Ngaoundéré,

Cameroun

b Programme agro-alimentaire, Cirad-amis,

73 rue J.-F. Breton, TA 40/16, 34398, Montpellier cedex 01,

France

${ }^{c}$ Ensia-Siarc,

1101 avenue d'Agropolis,

BP 5098 ,

34033, Montpellier cedex 01,

France

\section{Behavior of certain tropical fruits during the dewatering-impregnation soaking process in sucrose solutions.}

Abstract - Introduction. Kinetics of the dewatering-impregnation soaking process (DISP) of three tropical fruits - papayas (Carica papaya), mangoes (Mangifera indica) and bananas (Musa acuminata) - were compared. Materials and methods. All the experiments were carried out in a thermostated water bath system, with an initial sucrose concentration at $50 \mathrm{~g}$ sucrose $\times 100 \mathrm{~g} \mathrm{~g}^{-1}$ soaking solution and temperatures varying between 30 and $60{ }^{\circ} \mathrm{C}$. Fruit was cut into pieces of $9 \mathrm{~mm}$ in thickness and $31 \mathrm{~mm}$ in diameter. Fruit behavior in DISP was characterized by water loss, solute intake and loss of fruit solutes over $300 \mathrm{~min}$. A [fruit/ solution] ratio of [1/6.5] allowed fruit solute transfers (vitamin, mineral salts, acids) toward the soaking solution to be evaluated by direct measurements of vitamin concentration, conductivity and $\mathrm{pH}$ of the solution. Results and discussion. The lower the initial sugar content of fruit, the higher the solute gain. For the three fruits and for the operating conditions tested, losses in vitamin $\mathrm{C}$ evaluated from concentrated solution were less than $5 \mathrm{mg} \times 100 \mathrm{~g}^{-1}$ initial product. Those losses were most important during mango treatment. Finally, it was shown that an increase of temperature favored mass transfers.

Cameroon / Carica papaya (fruits) / Mangifera indica (fruits) / Musa acuminata (fruits) / conservation (storage) / dipping / sucrose / osmotic dehydration / diffusion / ascorbic acid / mineral content / moisture content
*Correspondance et tirés à part

Reçu le 7 juillet 2000 Accepté le 30 novembre 2000

Fruits, 2001, vol. 56, p. 75-83 (C) 2001 Cirad/EDP Sciences All rights reserved

Resumen Español, p. 83

\section{Comportement de certains fruits tropicaux traités par déshydratation- imprégnation par immersion dans une solution de saccharose.}

Résumé - Introduction. Les comportements de trois fruits tropicaux - papaye (Carica papaya), mangue (Mangifera indica) et banane (Musa acuminata) - traités par déshydratation-imprégnation par immersion (DII) en solutions concentrées de saccharose ont été comparés. Matériel et méthodes. Les cinétiques ont été conduites en bain thermostaté, avec des températures variant de 30 à $60{ }^{\circ} \mathrm{C}$ et une concentration initiale de la solution d'immersion fixée à $50 \mathrm{~g}$ de saccharose $\times 100 \mathrm{~g}^{-1}$ de solution. Les fruits ont été parés puis découpés en disques de $9 \mathrm{~mm}$ d'épaisseur et $31 \mathrm{~mm}$ de diamètre. Le comportement des fruits en DII a été caractérisé par la perte d'eau, le gain en solutés issus de la solution et la perte en solutés propres (vitamines, sels minéraux) du fruit pendant $300 \mathrm{~min}$. Le rapport [fruits/ solution] de $[1 / 6,5]$ a permis d'évaluer le transfert de solutés (vitamines, sels minéraux, acides) du fruit vers la solution, gràce à des mesures directes de teneur en vitamine, conductivité et pH. Résultats et discussion. Le gain en soluté a été d'autant plus important que la teneur initiale du fruit en sucre était faible. Pour les trois fruits et pour les conditions opératoires testées, les pertes en vitamine $C$ estimées à partir de la solution de déshydratation ont été inférieures à $5 \mathrm{mg} \times 100 \mathrm{~g}^{-1}$ de produit initial. Ces pertes ont été plus importantes lors du traitement des mangues. Enfin, l'augmentation de la température a favorisé les transferts de matière.

Cameroun / Carica papaya (fruit) / Mangifera indica (fruit) / Musa acuminata (fruit) / conservation (stockage) / immersion / saccharose / déshydratation osmotique / diffusion / acide ascorbique / teneur en éléments minéraux / teneur en eau 


\section{Introduction}

Les fruits et légumes sont une source importante de vitamines, éléments minéraux et fibres, substances indispensables pour la diète humaine [1-3]. En Afrique, au sud du Sahara, leur surproduction sur des périodes très courtes conduit à de fortes pertes après récoltes.

Pour faire face à la production saisonnière des fruits dans ces pays, plusieurs méthodes artisanales ou industrielles ont été développées qui permettent la conservation des récoltes : transformation en confiture, séchage, congélation... Toutefois, ces traitements sont généralement onéreux et ils diminuent la qualité nutritionnelle et organoleptique du produit [4]. Des prétraitements bien choisis permettent de mieux préserver les qualités initiales du fruit et de diminuer le coût du procédé de conservation complémentaire utilisé. L'une de ces méthodes est la déshydratation-imprégnation par immersion (DII) ou «déshydratation-osmotique ».

La DII exploite la propriété des aliments solides, riches en eau, de se déshydrater et d'incorporer les solutés de la solution fortement concentrée dans laquelle ils sont immergés. Cette incorporation, lorsqu'elle est faite avec certains solutés spécifiques, leur confère une texture souple proche de celle du fruit frais. Un contrôle approprié des paramètres intervenant dans le procédé permet d'orienter les transferts vers deux régimes de fonctionnement distincts : un régime de déshydratation caractérisé par une perte d'eau supérieure au gain en solutés et un régime d'imprégnation caractérisé par un gain en solutés supérieur à la perte en eau [5]. Une perte de solutés propres du produit peut également être, de plus, observée ; elle est quantitativement négligeable par rapport aux deux transferts précédents, mais essentielle quant aux qualités organoleptiques (l'acidité par exemple) et nutritionnelles (teneurs en vitamines et éléments minéraux) du produit obtenu [6-8]. Pendant la DII, les transferts sont isothermes et il n'y a pas de changement de phase. De plus, l'opération se déroulant à l'abri de l'oxygène de l'air, les phénomènes d'oxydation sont limités. L'étape préliminaire de la DII permet ainsi, du fait de la perte en eau de l'aliment, de diminuer la durée de séchage ultérieur appliqué au produit ainsi que la charge du séchoir, du congélateur ou de tout autre équipement de conservation complémentaire $[9,10]$. Pour les fruits, le traitement par DII est beaucoup plus approprié aux produits charnus qu'à d'autres qui le sont moins, tels que les agrumes qui, lors de leur préparation pour le traitement, subissent des lésions de membranes, un changement de texture et une perte importante de jus.

Sur le plan industriel, semi-industriel ou artisanal, le développement du procédé de DII se heurte généralement à une connaissance insuffisante du comportement du produit. L'objectif de ce travail a donc été d'observer l'évolution de certaines caractéristiques de trois fruits tropicaux communs - la banane, la mangue et la papaye - pendant un tel traitement. Ainsi, l'étude a cherché à évaluer l'influence de la nature du fruit et de la température sur les cinétiques de perte d'eau, de gain en soluté et de perte en vitamine $\mathrm{C}$, ainsi que la variation du $\mathrm{pH}$ et de la conductivité électrique de la solution d'immersion au cours du procédé.

\section{Matériel et méthodes}

\subsection{Matériel végétal}

Les fruits étudiés au cours de ce travail - banane, mangue et papaye - ont été achetés sur un marché local à Ngaoundéré au Cameroun. Certaines de leurs caractéristiques physicochimiques ont alors été mesurées avant tout traitement, à partir de l'observation de dix fruits par espèce considérée (tableau I).

Après lavage, puis trempage de $30 \mathrm{~min}$ dans une solution aqueuse d'hypochlorite de sodium à $0,08 \%$ de chlore actif, les fruits à traiter ont été rincés à l'eau courante, égouttés, parés, puis découpés en disques de $31 \mathrm{~mm}$ de diamètre et $9 \mathrm{~mm}$ d'épaisseur. 


\begin{abstract}
Tableau I.
Caractéristiques moyennes de bananes, mangues et papayes avant traitement par déshydratation-imprégnation par immersion dans une solution de saccharose.
\end{abstract}

\begin{tabular}{lccccc} 
Fruit étudié & $\begin{array}{c}{ }^{\circ} \text { Brix } \\
\left(\mathrm{g} \times 100 \mathrm{~g}^{-1}\right)\end{array}$ & $\begin{array}{c}\text { Humidité } \\
\left(\mathrm{g} \times 100 \mathrm{~g}^{-1} \mathrm{mf}\right)\end{array}$ & $\mathrm{pH}$ & $\begin{array}{c}\text { Cendres } \\
\left(\mathrm{g} \times 100 \mathrm{~g}^{-1} \mathrm{mf}\right)\end{array}$ & $\begin{array}{c}\text { Acide ascorbique } \\
\left(\mathrm{mg} \times 100 \mathrm{~g}^{-1} \mathrm{mf}\right)\end{array}$ \\
\hline Banane & 20,2 & 78,1 & 5,2 & 0,68 & 9 \\
Mangue & 13,1 & 86,1 & 4,7 & 0,62 & 42 \\
Papaye & 9,3 & 89,8 & 3,7 & 0,44 & 52
\end{tabular}

mf : matière fraîche.

\subsection{Procédures opératoires}

L'étude cinétique de DII a été conduite à température constante dans un bain thermostaté.

Des fioles de $800 \mathrm{~mL}$ contenant une solution de saccharose donnée ont été placées dans un bain-marie à $60^{\circ} \mathrm{C}$. Dès stabilisation de la température de la solution, les disques de fruits ont été introduits dans les fioles, à raison de $70 \mathrm{~g}$ de fruits par fiole environ, et un rapport massique moyen [fruits / solution] de [1 / 6,5]. Dix durées d'expérimentation ont été testées : $0,15,30$, $45,60,100,150,200,250$ et $300 \mathrm{~min}$, chacune d'elles étant associée à l'une des fioles utilisées. Les fioles correspondant aux temps d'expérimentation les plus longs ont été remplies les premières. Les fruits ont été maintenus immergés dans la solution sucrée concentrée à l'aide d'une grille fixée sur le couvercle de chaque pot.

Les fioles ont été agitées manuellement toutes les $5 \mathrm{~min}$.

\subsection{Mesures et calculs}

\subsubsection{Mesure des caractéristiques}

La teneur en sucre des fruits et des solutions de saccharose a été mesurée au réfractomètre et exprimée en degré Brix. Le $\mathrm{pH}$ des fruits a été mesuré au pH-mètre après broyage des fruits. La conductivité et le $\mathrm{pH}$ des solutions de saccharose ont été mesurés pour chacune des fioles en fin d'expérimentation. Les teneurs en matières sèches et en cendres des échantillons ont été déterminées selon les méthodes décrites dans les normes AOAC [11]. La vitamine C a été dosée par la méthode volumétrique au 2,6-dichloro-indophénol [12].

Chaque expérience a été répétée deux fois. Les écarts entre résultats issus de ces répétitions ont été inférieurs à $5 \%$.

\subsubsection{Calculs}

En considérant les différents transferts qui ont lieu au cours du procédé de DII (figure 1), il est possible d'effectuer le bilan des matières affectant le produit par suite des mouvements d'eau et de sucre, cela en utilisant certains paramètres adaptés à l'étude (tableau II).

\subsubsection{Bilan d'eau}

La masse d'eau de l'échantillon à l'instant t, ou $\left[\mathrm{M}_{\mathrm{e}}(\mathrm{t})\right]$ correspond à la différence entre la masse d'eau initiale, ou $\left[\mathrm{M}_{\mathrm{e}}\left(\mathrm{t}_{0}\right)\right]$ et la masse d'eau perdue du temps $t_{0}$ au temps $t$, ou $\left[P E(t) \times M_{p}\left(t_{0}\right)\right]$.

Or

et

$$
\left[\mathrm{M}_{\mathrm{e}}(\mathrm{t})=\mathrm{H}(\mathrm{t}) \times \mathrm{M}_{\mathrm{p}}(\mathrm{t})\right]
$$

d'où

$$
\left[M_{e}\left(t_{0}\right)=H\left(t_{0}\right) \times M_{p}\left(t_{0}\right)\right]
$$$$
\mathbb{M}_{e}(t)=\left[H\left(t_{0}\right) \times M_{p}\left(t_{0}\right]-\left[P E(t) \times M_{p}\left(t_{0}\right)\right]\right\} .
$$

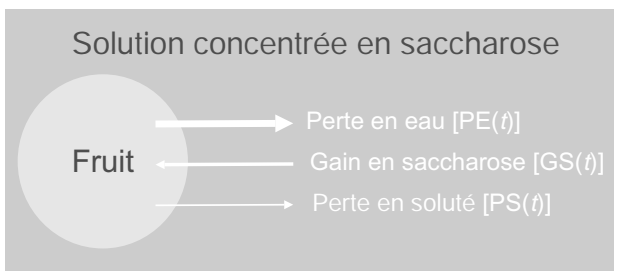

Figure 1.

Représentation schématique des transferts de matière au cours du traitement d'échantillons de fruit par déshydratation-imprégnation par immersion dans une solution concentrée de saccharose. 


\subsubsection{Bilan de sucre}

La masse de sucre de l'échantillon à l'instant $\mathrm{t}$, ou [S(t)] correspond au cumul de la masse de sucre initiale, ou $\left[S\left(t_{0}\right)\right]$ et de la masse de sucre imprégnée de l'instant $t_{0}$ à l'instant $\mathrm{t}$, ou $\left[\mathrm{GS}(\mathrm{t}) \times \mathrm{M}_{\mathrm{p}}\left(\mathrm{t}_{0}\right)\right]$, donc

$$
\left\{\mathrm{S}(\mathrm{t})=\mathrm{S}\left(\mathrm{t}_{0}\right)+\left[\mathrm{GS}(\mathrm{t}) \times \mathrm{M}_{\mathrm{p}}\left(\mathrm{t}_{0}\right)\right]\right\} .
$$

En considérant que le jus du fruit n'est constitué que de sucre et d'eau,

$$
\begin{gathered}
\left.\mathbb{B}\left(\mathrm{t}_{0}\right)=\mathrm{S}\left(\mathrm{t}_{0}\right) /\left[\mathrm{Me}\left(\mathrm{t}_{0}\right)+\mathrm{S}\left(\mathrm{t}_{0}\right)\right]\right\}, \\
\text { d'où } \\
\left\{\mathrm{S}\left(\mathrm{t}_{0}\right)=\left[\mathrm{B}\left(\mathrm{t}_{0}\right) \times \mathrm{Me}\left(\mathrm{t}_{0}\right)\right] /\left[1-\mathrm{B}\left(\mathrm{t}_{0}\right)\right]\right\} .
\end{gathered}
$$

\subsubsection{Bilan global}

Le bilan global, à l'instant $\mathrm{t}$, des mouvements de matières affectant le produit est la résultante du gain en saccharose, de la perte d'eau et de la perte en soluté, d'où :

$$
\begin{aligned}
\mathbb{M}_{\mathrm{p}}(\mathrm{t}) & =\mathrm{M}_{\mathrm{p}}\left(\mathrm{t}_{0}\right)+\left[\mathrm{GS}(\mathrm{t}) \times \mathrm{M}_{\mathrm{p}}\left(\mathrm{t}_{0}\right)\right] \\
& \left.-\left[\mathrm{PE}(\mathrm{t}) \times \mathrm{M}_{\mathrm{p}}\left(\mathrm{t}_{0}\right)\right]-\left[\mathrm{PS}(\mathrm{t}) \times \mathrm{M}_{\mathrm{p}}\left(\mathrm{t}_{0}\right)\right]\right\} .
\end{aligned}
$$

Il s'ensuit que

$$
\left.\left\{\mathrm{M}_{\mathrm{p}}\left(\mathrm{t}_{0}\right)-\mathrm{M}_{\mathrm{p}}(\mathrm{t})\right] / \mathrm{M}_{\mathrm{p}}\left(\mathrm{t}_{0}\right)=\mathrm{PE}(\mathrm{t})+\mathrm{PS}(\mathrm{t})-\mathrm{GS}(\mathrm{t})\right\} \text {. }
$$

La quantité $\left.\left\{M_{p}\left(t_{0}\right)-M_{p}(t)\right] / M_{p}\left(t_{0}\right)\right\}$ représente la réduction de poids $\mathrm{RP}(\mathrm{t})$ de l'échantillon, à l'instant $t$.

Les valeurs de perte en soluté [PS(t)] des fruits, obtenues au cours des cinétiques, étant relativement très faibles comparées à celles de gain en soluté, de réduction de poids ou de perte d'eau, cette quantité a été négligée dans la suite des calculs. Étant donné que l'accroissement de la fermeté des fruits en cours d'opération rend difficile l'extraction de jus, seul le degré Brix initial des fruits a été mesuré au réfractomètre. La valeur des teneurs en sucre des échantillons au cours du temps a ensuite été obtenue par calcul.

Finalement, il est possible d'écrire les expressions suivantes :

$$
\begin{aligned}
\mathrm{PE}(\mathrm{t}) & \left.=\mathrm{H}\left(\mathrm{t}_{0}\right)-\left\{\mathrm{M}_{\mathrm{p}}(\mathrm{t}) / \mathrm{M}_{\mathrm{p}}\left(\mathrm{t}_{0}\right)\right] \times \mathrm{H}(\mathrm{t})\right\} \\
\mathrm{RP}(\mathrm{t}) & =\left[\mathrm{M}_{\mathrm{p}}\left(\mathrm{t}_{0}\right)-\mathrm{M}_{\mathrm{p}}(\mathrm{t})\right] / \mathrm{M}_{\mathrm{p}}\left(\mathrm{t}_{0}\right) \\
\mathrm{GS}(\mathrm{t}) & =\mathrm{PE}(\mathrm{t})-\mathrm{RP}(\mathrm{t}) \\
\mathrm{S}(\mathrm{t}) & \left.=\left\{\mathrm{B}\left(\mathrm{t}_{0}\right) \times \mathrm{M}_{\mathrm{p}}\left(\mathrm{t}_{0}\right) \times \mathrm{H}\left(\mathrm{t}_{0}\right)\right] /\left[1-\mathrm{B}\left(\mathrm{t}_{0}\right)\right]\right\} \\
& +\left[\mathrm{GS}(\mathrm{t}) \times \mathrm{M}_{\mathrm{p}}\left(\mathrm{t}_{0}\right)\right] \\
\mathrm{B}(\mathrm{t}) & =\mathrm{S}(\mathrm{t}) /\left[\mathrm{M}_{\mathrm{p}}(\mathrm{t}) \times \mathrm{H}(\mathrm{t}) \times \mathrm{S}(\mathrm{t})\right]
\end{aligned}
$$

Sur une période donnée, le calcul de la pente de la droite, obtenue en rejoignant le premier et le dernier point expérimental, a permis d'évaluer l'importance des évolutions des paramètres du procédé.

\subsection{Influence de la température sur l'évolution des paramètres}

L'effet de trois températures $\left(30{ }^{\circ} \mathrm{C}, 50{ }^{\circ} \mathrm{C}\right.$ et $60^{\circ} \mathrm{C}$ ) a été comparé sur l'évolution des caractéristiques des fruits placés dans une solution de saccharose à $50^{\circ}$ Brix. La valeur des paramètres a été évaluée après $5 \mathrm{~h}$ de traitement par DII.

\section{Résultats et discussion}

Les valeurs de teneur en sucre, d'humidité et de cendres mesurées pour les trois fruits en début d'expérimentation ont été comparables aux données publiées par Ihekoronye et Ngoddy [3].

\subsection{Bilan des transferts dans les fruits}

\subsubsection{Perte d'eau}

Dans une solution de saccharose à $50^{\circ}$ Brix de concentration initiale, les échantillons de mangue, papaye et banane étudiés ont présenté sensiblement la même vitesse initiale de perte d'eau, en moyenne de $6,3 \times 10^{-3} \mathrm{~g}$ eau $\times g^{-1}$ fruit $\times \min ^{-1}$ (figure 2).

Par la suite, les vitesses instantanées de perte d'eau des tissus ont été décroissantes. Entre 200 et 300 min d'immersion, la perte d'eau des échantillons a été presque linéaire et les vitesses moyennes correspondantes ont été de $7,6 \times 10^{-4} \mathrm{~g}$ eau $\times \mathrm{g}^{-1}$ fruit $\times \mathrm{min}^{-1}$ pour la mangue, contre $2,3 \times 10^{-4}$ pour la papaye et $4,0 \times 10^{-4}$ pour la banane. La perte d'eau finale la plus importante a été obtenue par la mangue $\left(44,6 \mathrm{~g} \times 100 \mathrm{~g}^{-1}\right)$; celle de la papaye a été de $36,3 \mathrm{~g} \times 100 \mathrm{~g}^{-1}$ et celle de la banane de 28,8 $\mathrm{g} \times 100 \mathrm{~g}^{-1}$. La perte d'eau des fruits au cours du procédé de DII n’apparaît pas corrélée à leur teneur en eau initiale. 


\subsubsection{Gain en soluté}

Les vitesses initiales d'incorporation de soluté ont été comprises entre $6,7 \times 10^{-4}$ et $3,5 \times 10^{-3} \mathrm{~g}$ soluté $\times \mathrm{g}^{-1}$ fruit $\times \mathrm{min}^{-1}$, selon le fruit considéré, tandis que les vitesses moyennes entre 200 et 300 min d'expérimentation ont varié de $1,5 \times 10^{-4}$ à $2,0 \times$ $10^{-4} \mathrm{~g}$ soluté $\times \mathrm{g}^{-1}$ fruit $\times \mathrm{min}^{-1}$ (figure 3 ). Les gains finaux en solutés ont été de $19,2 \mathrm{~g} \times 100 \mathrm{~g}^{-1}$ fruit pour la papaye, $12 \mathrm{~g} \times$ $100 \mathrm{~g}^{-1}$ fruit pour la mangue et $8,5 \mathrm{~g} \times 100 \mathrm{~g}^{-1}$ fruit pour la banane. Au regard des données du tableau I, le gain en soluté en fin de procédé semble inversement proportionnel à la teneur initiale en sucres des fruits.

\subsubsection{Bilan des transferts d'eau et de soluté}

Globalement, les transferts opposés d'eau et de soluté ont conduit à une réduction de poids. Après 300 min d'expérimentation, ces pertes ont été de $32,6 \mathrm{~g} \times 100 \mathrm{~g}^{-1}$ fruit pour la mangue, $20,3 \times 100 \mathrm{~g}^{-1}$ fruit pour la banane et $17,1 \times 100 \mathrm{~g}^{-1}$ fruit pour la papaye (figure 4).

L'incorporation de saccharose au cours du procédé conduit à des valeurs de degré Brix finales presque identiques pour la papaye $\left(34,4^{\circ}\right.$ Brix), la mangue $\left(36^{\circ}\right.$ Brix $)$ et la banane $\left(35,9^{\circ}\right.$ Brix) (figure 5). Des résultats similaires avaient été trouvés par Machado Martinez [13] lors du traitement de cubes d'ananas, mangues et papayes.

\section{2. Évolution des caractéristiques de la solution d'immersion}

L'évolution au cours du temps du $\mathrm{pH}$, de la conductivité et de la teneur en vitamine $\mathrm{C}$ de la solution traduit la diffusion des constituants solubles et de l'eau dans le sens «fruit » vers «solution».

\subsection{1. Évolution du pH}

Le $\mathrm{pH}$ de la solution a diminué au cours du temps (figure 6). En fin de procédé, la variation du $\mathrm{pH}$ de la solution a atteint 1,2 unités pour la banane, 1,6 unités pour la mangue et 2,6 unités pour la papaye. Plus le fruit est acide (tableau I), plus le $\mathrm{pH}$

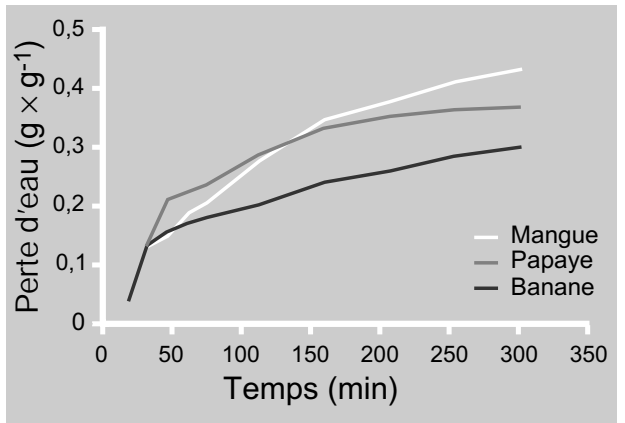

\section{Figure 2.}

Évolution des pertes d'eau pour des disques

(31 $\mathrm{mm}$ de diamètre / $9 \mathrm{~mm}$ ) de banane, mangue et papaye, pendant un traitement par déshydratationimprégnation par immersion dans une solution de saccharose à $50^{\circ} \mathrm{Brix}$, et à $60^{\circ} \mathrm{C}$.

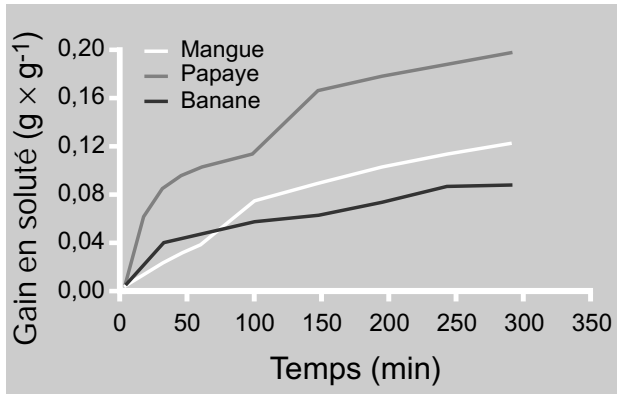

\section{Figure 3.}

Évolution des gains en soluté pour des disques

(31 $\mathrm{mm}$ de diamètre $/ 9 \mathrm{~mm}$ ) de banane, mangue et papaye, pendant un traitement par déshydratationimprégnation par immersion dans une solution de saccharose à $50^{\circ} \mathrm{Brix}$ et à $60^{\circ} \mathrm{C}$.

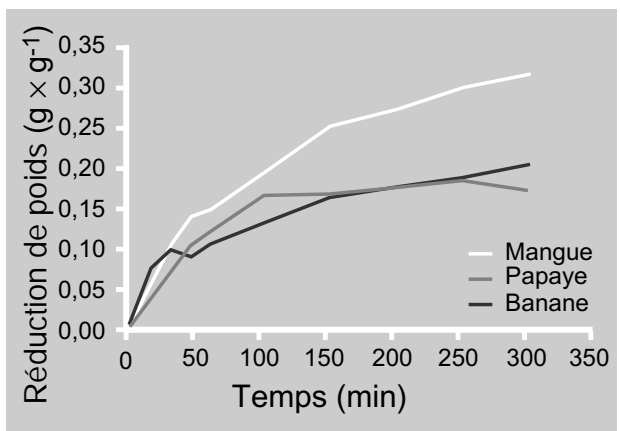

\section{Figure 4.}

Évolution des réductions de poids pour des disques (31 $\mathrm{mm}$ de diamètre $/ 9 \mathrm{~mm}$ ) de banane, mangue et papaye, pendant un traitement par déshydratationimprégnation par immersion dans une solution de saccharose à $50^{\circ} \mathrm{Brix}$, et à $60^{\circ} \mathrm{C}$.

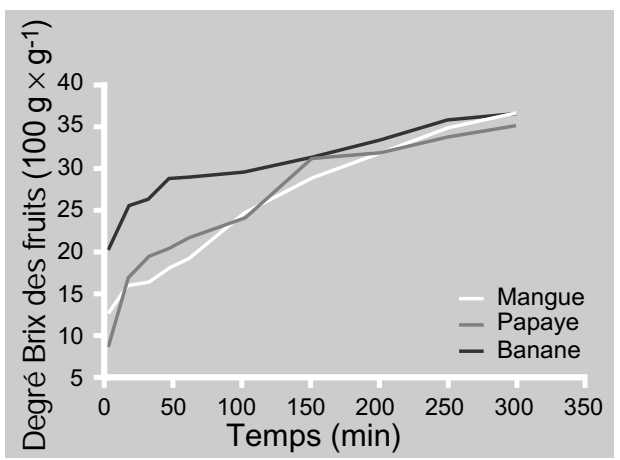

\section{Figure 5.}

Évolution des degrés Brix calculés pour des disques (31 $\mathrm{mm}$ de diamètre $/ 9 \mathrm{~mm}$ ) de banane, mangue et papaye, pendant un traitement par déshydratation-imprégnation par immersion dans une solution de saccharose à $50^{\circ}$ Brix, et à $60^{\circ} \mathrm{C}$. de la solution a varié. L'acidité des fruits naturellement très acides sera donc adoucie en fin de DII. Jiokap Nono et al. [14] ont 
Figure 6.

Évolution du pH de la solution d'immersion de disques (31 mm de diamètre / $9 \mathrm{~mm}$ ) de banane, mangue et papaye, pendant un traitement par déshydratationimprégnation par immersion dans une solution de saccharose à $50^{\circ} \mathrm{Brix}$, et à $60^{\circ} \mathrm{C}$.

Figure 7.

Évolution des pertes en vitamine $C$ pour des disques (31 $\mathrm{mm}$ de diamètre / $9 \mathrm{~mm}$ ) de banane, mangue et papaye, pendant un traitement par déshydratationimprégnation par immersion dans une solution de saccharose à $50^{\circ}$ Brix, et à $60^{\circ} \mathrm{C}$.
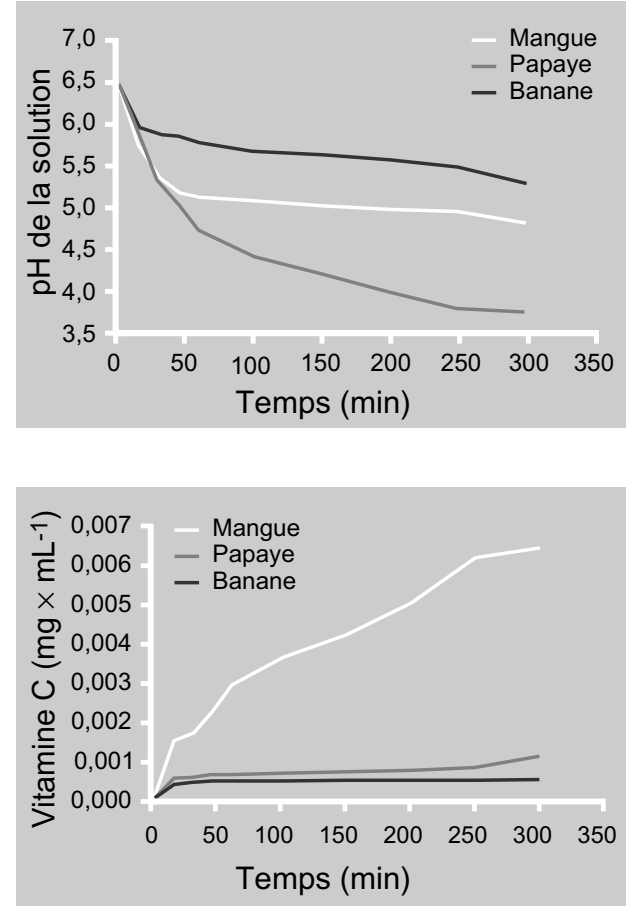

montré que le recyclage de la solution permettait de limiter le transfert d'acides du produit vers la solution car la différence de concentration en acides est plus faible entre une solution vieillie et les fruits. En revanche, en présence de produits très acides, l'acidification de la solution peut conduire à une hydrolyse du saccharose en glucose et fructose. À la surface du produit, ces sucres peuvent entraîner, par brunissement non enzymatique, un changement de couleur notable, notamment pendant l'étape de séchage qui suivra éventuellement.

\subsection{2. Évolution de la teneur en vitamine $C$}

La perte des fruits en vitamine $\mathrm{C}$ a été plus importante pour la mangue que pour la banane et la papaye où elle a été presque négligeable (figure 7). Ces résultats montrent que la perte en vitamine $C$ ne serait pas directement liée à sa teneur initiale dans le fruit. Toutefois, pour les conditions expérimentales testées, la perte en vitamine $C$ du fruit, due à la diffusion de cet élément à l'intérieur de la solution, est restée inférieure à $0,007 \mathrm{mg} \times \mathrm{mL}^{-1}$. En tenant compte du rapport massique [fruits / solution] utilisé, cette valeur correspond à une perte en vitamine $C$ de 4,26 mg $\times 100 \mathrm{~g}^{-1}$ de produit initial, soit moins de $10 \%$ de la teneur initiale du fruit en vitamine C. Compte tenu de la température de travail $\left(60{ }^{\circ} \mathrm{C}\right)$ et des conditions de détermination des pertes en vitamine $\mathrm{C}$, estimées à partir de sa concentration dans la solution de déshydratation, ces pertes pourraient être sous-estimées. La diffusion de la vitamine $\mathrm{C}$ au cours du procédé de DII pourrait être réduite, comme dans le cas de celle des acides, par recyclage de la solution.

\section{Tableau II.}

Notation et signification des paramètres utilisés pour évaluer le comportement d'échantillons de fruits immergés dans une solution de saccharose.

\begin{tabular}{|c|c|c|}
\hline Notation & Signification & Unité \\
\hline $\mathrm{PE}(t)$ & Perte d'eau à l'instant $t$, relativement à la masse initiale du produit ; $0 \leq \mathrm{PE}<1$ & g/g produit initial \\
\hline $\mathrm{S}(t)$ & Masse de sucre dans l'échantillon à l'instant $t$ & g \\
\hline $\mathrm{GS}(t)$ & Gain en soluté à l'instant $t$, relativement à la masse initiale du produit ; $0 \leq \mathrm{GS}<1$ & $\mathrm{~g} / \mathrm{g}$ produit initial \\
\hline $\mathrm{RP}(t)$ & Réduction de poids à l'instant $t$, relativement à la masse initiale du produit ; $0 \leq R P<1$ & g/g produit initial \\
\hline $\mathrm{PS}(t)$ & Perte en soluté à l'instant $t$, relativement à la masse initiale du produit ; $0 \leq \mathrm{PS}<1$ & $\mathrm{~g} / \mathrm{g}$ produit initial \\
\hline $\mathrm{B}(t)$ & Masse de sucre rapportée à la masse de jus dans l'échantillon à l'instant $t ; 0 \leq \mathrm{B}<1$ & $g / g$ \\
\hline $\mathrm{M}_{\mathrm{e}}(t)$ & Masse d'eau contenue dans l'échantillon à l'instant $t$ & $g$ \\
\hline $\mathrm{M}_{\mathrm{p}}(t)$ & Masse de l'échantillon à l'instant $t$ & g \\
\hline$H(t)$ & Teneur en eau de l'échantillon à l'instant $t$, relativement à la masse initiale du produit & $\mathrm{g} / \mathrm{g}$ produit initial \\
\hline$t$ & Temps & $\mathrm{s}$ \\
\hline
\end{tabular}




\subsection{3. Évolution de la conductivité de la solution}

$\mathrm{Au}$ cours de la DII, la conductivité de la solution a augmenté progressivement pour chacun des trois fruits étudiés (figure 8). Cependant, la plus forte variation a été observée pendant le traitement des échantillons de banane, supérieure à celle de la mangue, puis de la papaye. Les teneurs de ces fruits en sels minéraux étant de $0,68 \mathrm{~g} \times$ $100 \mathrm{~g}^{-1}$ pour la banane, $0,62 \mathrm{~g} \times 100 \mathrm{~g}^{-1}$ pour la mangue et $0,44 \mathrm{~g} \times 100 \mathrm{~g}^{-1}$ pour la papaye, la variation de conductivité de la solution après 300 min de traitement serait d'autant plus grande que la teneur en cendres des fruits est plus élevée. Cette perte en minéraux au cours du procédé ne peut être évitée lorsque la solution de déshydratation est chaque fois renouvelée. Cependant, comme l'a montré Valdez Fragoso [15] lors du traitement de pommes, l'utilisation d'un sirop vieilli permettrait de préserver ces constituants du fruit.

\subsection{Influence de la température}

D'une manière générale, l'augmentation de la température de traitement des fruits a accéléré l'évolution des paramètres du

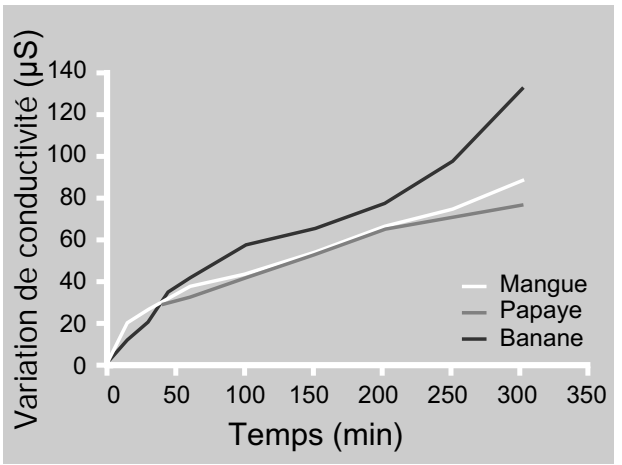

Figure 8.

Évolution de la variation de conductivité de la solution d'immersion de disques (31 $\mathrm{mm}$ de diamètre $/ 9 \mathrm{~mm}$ ) de banane, mangue et papaye, pendant un traitement par déshydratationimprégnation par immersion dans une solution de saccharose à $50^{\circ} \mathrm{Brix}$, et à $60^{\circ} \mathrm{C}$ procédé (tableau III). La perte d'eau, la réduction de poids et le gain en solutés des fruits, ainsi que les variations de $\mathrm{pH}$ et de conductivité de la solution ont régulièrement augmenté avec la température.

\section{Conclusion}

Pour les échantillons de banane, mangue et papaye traités par un procédé de déshydratation-imprégnation par immersion au cours de nos travaux, il y a eu perte d'eau, réduction de poids et gain en solutés.

\section{Tableau III.}

Caractéristiques finales d'échantillons de banane, mangue et papaye après 300 min de traitement par déshydratation-imprégnation par immersion dans une solution de saccharose initialement à $50{ }^{\circ}$ Brix, en fonction de trois températures d'expérimentation.

\begin{tabular}{|c|c|c|c|c|c|c|c|c|}
\hline Fruit étudié & $\begin{array}{c}\text { Température } \\
\left({ }^{\circ} \mathrm{C}\right)\end{array}$ & $\begin{array}{l}\text { Perte d'eau } \\
\left(\mathrm{g} \times 100 \mathrm{~g}^{-1}\right)\end{array}$ & $\begin{array}{c}\text { Gain } \\
\text { en solutés } \\
\left(\mathrm{g} \times 100 \mathrm{~g}^{-1}\right)\end{array}$ & $\begin{array}{l}\text { Réduction } \\
\text { de poids } \\
\left(\mathrm{g} \times 100 \mathrm{~g}^{-1}\right)\end{array}$ & $\begin{array}{c}{ }^{\circ} \text { Brix } \\
\left(\mathrm{g} \times 100 \mathrm{~g}^{-1}\right)\end{array}$ & $\mathrm{pH}$ & $\begin{array}{c}\text { Variation } \\
\text { de conductivité } \\
(\mu S)\end{array}$ & $\begin{array}{l}\text { Vitamine C } \\
\text { dans la solution } \\
\left(\mathrm{g} \times \mathrm{mL}^{-1}\right)\end{array}$ \\
\hline \multirow[t]{3}{*}{ Banane } & 30 & 16,0 & 6,0 & 10,0 & 28,6 & 5,55 & 50 & $<0,0005$ \\
\hline & 50 & 25,7 & 9,6 & 16,1 & 34,9 & 5,39 & 111 & $<0,0005$ \\
\hline & 60 & 28,8 & 8,5 & 20,3 & 35,9 & 5,33 & 130 & $<0,0005$ \\
\hline \multirow[t]{3}{*}{ Mangue } & 30 & 34,0 & 7,0 & 27,0 & 27,3 & 5,75 & 32 & 0,0022 \\
\hline & 50 & 42,0 & 10,0 & 32,0 & 34,2 & 4,85 & 76 & 0,0038 \\
\hline & 60 & 44,6 & 12,0 & 32,6 & 36,0 & 4,90 & 87 & 0,0066 \\
\hline \multirow[t]{3}{*}{ Papaye } & 30 & - & - & - & - & - & - & - \\
\hline & 50 & 29,1 & 16,2 & 12,9 & 29,8 & 4,65 & 42 & 0,001 \\
\hline & 60 & 36,3 & 19,2 & 17,1 & 34,4 & 3,85 & 75 & 0,001 \\
\hline
\end{tabular}


La perte d'eau maximale a été observée avec les rondelles de mangue $(44,6 \%$ à $60{ }^{\circ} \mathrm{C}$ ) et la plus faible avec les rondelles de banane $\left(16 \%\right.$ à $\left.30{ }^{\circ} \mathrm{C}\right)$.

Dans nos conditions opératoires, la plus forte réduction de poids a été subie par la mangue $\left(32,6 \%\right.$ à $\left.60{ }^{\circ} \mathrm{C}\right)$ et la plus faible par la papaye $\left(<12,9 \%\right.$ à $\left.30^{\circ} \mathrm{C}\right)$.

Les teneurs en sucre des fruits ont augmenté avec la durée du traitement et elles ont été directement liées aux gains en solutés des fruits. Pour des conditions opératoires données, le degré Brix final du fruit a semblé ne pas être dépendant de sa nature. De plus, une température du traitement plus élevée favoriserait les transferts de matière au cours du procédé.

Les évolutions de $\mathrm{pH}$ et de conductivité de la solution au cours du procédé de DII se sont révélées liées à la perte en substances solubles des fruits, dont la vitamine $\mathrm{C}$ et les sels minéraux.

Ainsi, d'après nos résultats, la mesure du degré Brix des fruits et celle de la conductivité et du $\mathrm{pH}$ de la solution d'immersion pourraient servir à mieux évaluer les caractéristiques organoleptiques du produit en fin d'application d'un traitement de DII à des fruits devant être conservés.

\section{Remerciements}

Ce travail a été soutenu par l'Agence universitaire de la francophonie (Aupelf-Uref) dans le cadre d'un projet du Fonds international de coopération universitaire (Ficu).

\section{Références}

[1] Woot-Tsuen Wu Leung, Food composition table for use in Africa, Food and Agriculture Organization of the United Nations, Roma, Italy, 1968

[2] Leroy J.F., Les fruits tropicaux et subtropicaux, valeur alimentaire, in: Les fruits tropicaux et subtropicaux, collection Que sais-je?, $n^{\circ}$ 237, PUF, Paris, 1968, pp. 7-15.

[3] Ihekoronye A.I., Ngoddy P.O., Chapter 19: Tropical fruits and vegetables, in: Integrated Food Science and Technology for the Tropics, Macmillan Publishers Ltd, London, UK, 1985, 293-311.
[4] Cheftel J.-C., Cheftel H., Agents et mécanismes de détérioration des aliments, in: Lavoisier (Ed.), Introduction à la biochimie et à la technologie des aliments, vol. 1, Technique et Documentation, Paris, 1977, 271-369.

[5] Raoult-Wack A.L., Recent advances in the osmotic dehydration of foods, Trends Food Sci. Tech. 5 (1994) 255-262.

[6] Dixon G.M., Jen J.J., Changes of sugars and acids of osmovac-dried apples slices, J. Food Sci. 42 (4) (1977) 1126-1127.

[7] Vial C., Guilbert S., Cuq J.L., Osmotic dehydration of kiwi fruits: influence of process variables on the color and ascorbic acid content, Sci. Aliment. 11 (1991) 63-84.

[8] Torregianni D., Osmotic dehydration in fruit and vegetable processing, Food Res. Int. 26 (1993) 59-68.

[9] Collignan A., Raoult-Wack A.L., Themelin A., Energy study of food processing by osmotic dehydration and air drying, Agr. Eng. J. 1 (3) (1992) 125-135.

[10] Themelin A., Optimisation des procédés combinés de déshydratation-imprégnation par immersion (dans des solutions concentrées) et de séchage par entraînement (à l'air chaud), thèse, Ensia, Massy, France, 1994.

[11] Anonymous, Official methods of analysis (13th ed.), Association of Official Analytical Chemists (AOAC), Washington, DC, USA, 1980.

[12] Audigie C., Figarella J., Zonszain F., Manipulation d'analyse biochimique, Doin (Ed.), Paris, France, 1980, 274 p.

[13] Machado Martinez J.M., Fabrication d'un mélange de fruits tropicaux par un procédé combiné de déshydratation-imprégantion par immersion suivi d'un séchage à l'air chaud, mémoire de mastère, Ensia-Siarc, Montpellier, France, 1994, 38 p.

[14] Jiokap Nono Y., Giroux F., Cuq B., RaoultWack A.L., Étude des paramètres de contrôle et de commande du procédé de déshydratation-imprégnation par immersion sur système probatoire automatisé : application au traitement des pommes Golden, Kapseu C., Kayem J. (Éds.), Séminaire-atelier international sur le séchage et sur la valorisation du karité et de l'aiélé, Ngaoundéré, Cameroun, 1999.

[15] Valdez Fragoso A., Étude du recyclage des solutions concentrées en déshydratationimprégnation par immersion. Application au cas du traitement de pommes dans une solution de saccharose, thèse, Ensia, Massy, France, 1998. 


\section{Comportamiento de algunos frutos tropicales tratados mediante deshidratación- impregnación por inmersión en una solución de sacarosa.}

Resumen - Introducción. Se comparó el comportamiento de tres frutas tropicales - papaya (Carica papaya), mango (Mangifera indica) y banano (Musa acuminata) -, tratadas por deshidratación-impregnación mediante inmersión en soluciones concentradas de sacarosa. Material y métodos. Se realizaron las cinéticas en baño termostático, con temperaturas que iban de 30 a $60{ }^{\circ} \mathrm{C}$ y una concentración inicial de la solución de inmersión fijada a $50 \mathrm{~g}$ de sacarosa $\times 100 \mathrm{~g}^{-1}$ de solución. Se pelaron y cortaron los frutos en rodajas de $9 \mathrm{~mm}$ de grosor y $31 \mathrm{~mm}$ de diámetro. El comportamiento de los frutos en DII se caracterizó por la pérdida de agua, la ganancia en solutos procedentes de la solución y la pérdida de solutos propios (vitaminas, sales minerales) del fruto durante $300 \mathrm{~min}$. La relación [frutos / solución] de [1 / 6,5] permitió evaluar la transferencia de solutos (vitaminas, sales minerales, ácidos) del fruto hacia la solución, gracias a medidas directas de contenido en vitamina, conductividad y $\mathrm{pH}$. Resultados y discusión. La ganancia en solutos fue más significativa puesto que el contenido inicial del fruto en azúcar era bajo. En los tres frutos y en las condiciones de operación probadas, las pérdidas en vitamina $C$ estimadas a partir de la solución de deshidratación fueron inferiores a $5 \mathrm{mg} \times 100 \mathrm{~g}^{-1}$ de producto inicial. Estas pérdidas fueron más importantes en el tratamiento de mangos. Por último, el aumento de la temperatura favoreció las transferencias de materia.

Camerùn / Carica papaya (fruto) / Mangifera indica (fruto) / Musa a cuminata (fruto) / conservación (almacenamiento) / inmersion / sacarosa / deshidratacion osmotica / difusion / acido ascorbico / contenido mineral / contenido de humedad 